\title{
Total-Body PET Imaging for up to 30 Days After Injection of ${ }^{89} \mathrm{Zr}$-Labeled Antibodies
}

\author{
Zachary T. Rosenkrans ${ }^{1}$ and Weibo Cai ${ }^{1,2}$ \\ ${ }^{1}$ Department of Pharmaceutical Sciences, University of Wisconsin-Madison, Madison, Wisconsin; and ${ }^{2}$ Departments of Radiology \\ and Medical Physics, University of Wisconsin-Madison, Madison, Wisconsin
}

\section{See the associated article on page 453.}

\section{A}

fter the continued efforts toward the development of PET scanners over the last several decades, a major milestone was achieved with the recent completion of total-body PET scanners. Both clinical and preclinical total-body PET scanners have been developed by the EXPLORER Consortium (1-3). These state-ofthe-art scanners hold many advantages over previously developed PET scanners. For example, PET scans can be performed much more quickly because of a larger field of view and higher sensitivity. In addition, PET scans can be performed much later after tracer injection. In many cases, whole-body dynamic PET imaging can also provide invaluable information that could not be attained before. Both the clinical and the preclinical total-body PET scanners hold tremendous potential for various future biomedical applications, and the scientific community is looking forward to what can be done in the immediate future to unleash their full potential.

In this issue of The Journal of Nuclear Medicine, Berg et al. report PET imaging of rhesus monkeys with the primate miniEXPLORER scanner (4). They compared 4 different tracers, all ${ }^{89} \mathrm{Zr}$-labeled antibodies, and were able to acquire high-quality PET images for up to $30 \mathrm{~d}$ after tracer injection $(\sim 10$ decay half-lives of ${ }^{89} \mathrm{Zr}$ ). The antibody they used was a humanized monoclonal IgG antibody against glycoprotein $\mathrm{D}(\mathrm{gD})$, developed by Genentech, Inc.

${ }^{89} \mathrm{Zr}$-labeled antibodies have been studied for several decades, both preclinically and clinically (5). The commonly used chelator is desferrioxamine B (DFO), and various linkers have been investigated for better in vivo performance of ${ }^{89} \mathrm{Zr}$-based tracers. Since ${ }^{89} \mathrm{Zr}^{4+}$ prefers the formation of an octadentate complex, and the DFO chelator has only 6 oxygen atoms, such a combination is not ideal in terms of in vivo stability. Therefore, significant bone uptake has been observed at late time points in many preclinical studies. In clinical studies with ${ }^{89} \mathrm{Zr}$-labeled antibodies, bone uptake was not as obvious and did not seem to pose a significant concern. Nonetheless, substantial effort has been devoted to the

Received Oct. 2, 2019; revision accepted Dec. 2, 2019.

For correspondence or reprints contact: Weibo Cai, Departments of Radiology and Medical Physics, University of Wisconsin-Madison, Room 7137, 1111 Highland Ave., Madison, WI 53705

E-mail: wcai@uwhealth.org

Published online Dec. 5, 2019.

COPYRIGHT (c) 2020 by the Society of Nuclear Medicine and Molecular Imaging. DOI: 10.2967/jnumed.119.236166 development of better chelators for ${ }^{89} \mathrm{Zr}$. An octadentate variant of DFO, desferrioxamine* (DFO*), was developed which has an additional hydroxamate unit (6). With $\mathrm{DFO}^{*}$ as the chelator, ${ }^{89} \mathrm{Zr}$ had a lower signal in bone and liver than did DFO-based tracers (7). As such, DFO* is a promising chelator for future clinical translation of ${ }^{89} \mathrm{Zr}$-based PET tracers.

The principal aim of the work by Berg et al. (4) was to demonstrate the feasibility of extending nonhuman-primate ${ }^{89} \mathrm{Zr}$-based PET imaging studies to up to $30 \mathrm{~d}$ after injection with a primate mini-EXPLORER total-body PET system. Such extension was possible with this imaging system because of the increased sensitivity from a $45-\mathrm{cm}$ axial field of view and a $43.5-\mathrm{cm}$ bore diameter. The ability to image at such late time points could provide unprecedented biologic information about the long-term behavior of radiolabeled antibodies in vivo. In a secondary objective, the linker between the DFO or DFO* and the antibody, which plays an important role in the in vivo stability and behavior of ${ }^{89} \mathrm{Zr}$-labeled antibodies (8), was investigated. Two different chelators and 2 different linkers were used to label the antigD antibody. The 4 tracers (i.e., ${ }^{89} \mathrm{Zr}$-DFO-Bz-NCS-antigD, ${ }^{89} \mathrm{Zr}$-DFO-squaramide-antigD, ${ }^{89} \mathrm{Zr}$-DFO*-Bz-NCS-antigD, and ${ }^{89} \mathrm{Zr}$-DFO*-squaramide-antigD) were compared in rhesus monkeys over the 30 -d period after tracer injection to identify the best tracer for future investigation.

The feasibility of total-body PET at $30 \mathrm{~d}$ after injection of ${ }^{89} \mathrm{Zr}$ labeled antibodies was clearly demonstrated. Even with less than $40 \mathrm{kBq}$ of radioactivity in each rhesus monkey, PET images were obtained with sufficient quality to identify various organs of interest. The images at early time points were of excellent quality, with about $40 \mathrm{MBq}$ of radioactivity being injected into each approximately $3-\mathrm{kg}$ rhesus monkey. To compare the day 0 pharmacokinetics of the 4 tracers, dynamic PET scans during the first hour were performed. They revealed some interesting differences among the 4 tracers. Both tracers with the Bz-NCS linker group showed increased activity in the bladder toward the end of the 60min dynamic scan in comparison to both tracers with the squaramide linker groups. These findings suggest that the squaramide linker is significantly more stable in vivo than the commonly used Bz-NCS linker. Whether the chelator was DFO or DFO* did not appear to make a significant difference within the first hour of injection.

The whole-body radioactivity of each rhesus monkey was also measured over the course of $30 \mathrm{~d}$. Since all variables other than the chelator or linker used were consistent for the 4 PET tracers, the dramatic difference in whole-body radioactivity retention is quite interesting. This retention varied drastically from approximately $30 \%$ to more than $90 \%$ of the injected dose at the second time point (day 2 or 3). Enzyme-linked immunosorbent assay results showed that all $\mathrm{IgG}$ concentrations were essentially the same for all 4 
groups. Although there were some differences in the radiolabeling purities (i.e., greater impurities for the Bz-NCS groups), the authors suggest that the primary reason for this unexpected finding was that during the transportation of the radiolabeled antibodies, the high activity concentrations resulted in radiolytic degradation of the linker or chelator and such degradation occurred to a greater extent in the Bz-NCS groups than in the squaramide groups. These results suggest that in the future, greater care will need to be taken when transporting radiotracers, such as by ensuring proper temperature control. Additional quality control and purification of the tracers may also be needed at the PET facility before injection (which was not performed in this study) to ensure more accurate and meaningful PET imaging data, quantification, and result interpretation.

At late time points, the biodistribution and quantitative tracer uptake in the 4 groups were largely similar within each group of 3 primates. However, there were some notable differences among the 4 tracers, such as in whole-body retention and liver and bone uptake. The reason for this finding is unclear. One interesting aspect of this study is that tracer injection into the left brachial artery was administered after unlabeled antigD antibody $(10 \mathrm{mg} / \mathrm{kg})$ had been injected in the right brachial artery. Tracer injection into the artery in quite unusual and led to some radioactivity remaining at the injection site for several animals in the ${ }^{89} \mathrm{Zr}-\mathrm{DFO}-\mathrm{Bz}-\mathrm{NCS}-$ antigD and ${ }^{89} \mathrm{Zr}$-DFO-squaramide-antigD groups. The possibility that the remaining radioactivity may be partially responsible for the experimental findings needs to be investigated in future studies.

The results of this work are an important reminder that the choice of chelator is not trivial. The use of octadentate DFO* gave rise to fewer osteophilic catabolites of ${ }^{89} \mathrm{Zr}$-labeled antibodies and improved PET image signal at late time points, which is likely the result of a more stable complex with ${ }^{89} \mathrm{Zr}$ for the octadentate DFO* than for the hexadentate DFO. In addition, the squaramide linker may also provide some advantages over Bz-NCS in terms of shelf-life stability. Most importantly, the study demonstrated the tremendous potential of total-body PET scanners, which enabled unprecedented late-time-point imaging of ${ }^{89} \mathrm{Zr}$-labeled antibodies. Such long-term serial PET imaging could help answer many biologic questions about the in vivo behavior of radiolabeled antibodies (2). Many additional experiments to supplement the PET scans are needed to glean such biologic insight and validate the biologic relevance of the long-term PET imaging data, since the radioactivity at $30 \mathrm{~d}$ after injection might be largely dissociated from the antibody or drug, especially with a residualizing metal such as ${ }^{89} \mathrm{Zr}$. We look forward to future studies on this topic. With broader availability of both preclinical and clinical total-body PET scanners in the future, intensified scientific endeavors will be needed from research groups internationally. Such efforts will eventually lead to improved clinical translation and investigation of novel PET tracers and better patient care.

\section{DISCLOSURE}

Financial support was received from the University of WisconsinMadison and the National Institutes of Health (P30CA014520). No other potential conflict of interest relevant to this article was reported.

\section{REFERENCES}

1. Berg E, Zhang X, Bec J, et al. Development and evaluation of mini-EXPLORER: a long axial field-of-view PET scanner for nonhuman primate imaging. $J$ Nucl Med. 2018;59:993-998.

2. Cherry SR, Jones T, Karp JS, Qi J, Moses WW, Badawi RD. Total-body PET: maximizing sensitivity to create new opportunities for clinical research and patient care. J Nucl Med. 2018;59:3-12.

3. Badawi RD, Shi H, Hu P, et al. First human imaging studies with the EXPLORER total-body PET scanner. J Nucl Med. 2019;60:299-303.

4. Berg E, Gill H, Marik J, et al. Total-body PET and highly stable chelators together enable meaningful ${ }^{89} \mathrm{Zr}$-antibody PET studies up to 30 days after injection. $J$ Nucl Med. 2020;61:453-460.

5. Jauw YW, Menke-van der Houven van Oordt CW, Hoekstra OS, et al. Immunopositron emission tomography with zirconium-89-labeled monoclonal antibodies in oncology: what can we learn from initial clinical trials? Front Pharmacol. 2016; $7: 131$.

6. Patra M, Bauman A, Mari C, et al. An octadentate bifunctional chelating agent for the development of stable zirconium- 89 based molecular imaging probes. Chem Commun (Camb). 2014;50:11523-11525.

7. Vugts DJ, Klaver C, Sewing C, et al. Comparison of the octadentate bifunctional chelator DFO*-pPhe-NCS and the clinically used hexadentate bifunctional chelator DFO-pPhe-NCS for ${ }^{89} \mathrm{Zr}$-immuno-PET. Eur J Nucl Med Mol Imaging. 2017;44:286-295

8. Dilworth JR, Pascu SI. The chemistry of PET imaging with zirconium-89. Chem Soc Rev. 2018;47:2554-2571. 